\title{
Low Power Fast Settling Switched Capacitor PTAT Current Reference Cir- cuit for Low Frequency Applications
}

Muhammed Mansoor C. B Hanumantha Rao G., Rekha S.

Department of Electronics and Communication Engineering, National Institute of Technology Karnataka, Surathkal, 575025, India

A R T I CLE INFO

Article history:

Received: 29 August, 2020

Accepted: 06 November, 2020

Online: 27 November, 2020

Keywords:

PTAT

Switched Capacitor

Log-Domain Filter

\begin{abstract}
A B S T R A C T
This paper presents a low voltage, low power, fast settling switched capacitor based Proportional to Absolute Temperature (PTAT) current reference circuit. Unlike in a conventional resistor based PTAT current source, the proposed circuit saves a significant amount of silicon area on chip and hence the circuit becomes less susceptible to process variations. It creates a reference current of $1 \mathrm{nA}$ from a $0.5 \mathrm{~V}$ power supply at room temperature $\left(27^{\circ} \mathrm{C}\right)$. It has PTAT characteristics in the temperatures from $-10^{\circ} \mathrm{C}$ to $80^{\circ} \mathrm{C}$. The circuit draws a very low power of $1.5 \mathrm{nW}$ and exhibits a good supply voltage sensitivity of $3.2 \% / V$. A startup circuit connected to the PTAT source improves the transient response by reducing the settling time. To test the PTAT current reference circuit, a low power log-domain filter which can be used for biomedical applications is realised and biased with the proposed PTAT current source. Results show that the filter cutoff frequency is constant over temperature variations. The CMOS technology used for designing the circuits is UMC $65 \mathrm{~nm}$ and tool used for simulations is Cadence Virtuoso.
\end{abstract}

\section{Introduction}

Portable and wearable/implantable devices are gaining worldwide acceptance among consumer/biomedical electronics. Circuit designs in such devices have a bottleneck on power dissipation and chip area. Log-domain circuits are one of the best possibilities in exploring low power, high dynamic range analog designs. Log-domain filters and transconductance-capacitor $\left(G_{m}-C\right)$ filters can be used in biological data acquisition system which involves low frequency signals like ECG (electrocardiogram) and EEG (electroencephalogram). Since the transistors in these log-domain filters are working in sub-threshold mode of operation (weak-inversion), a low cutoff frequency can be achieved with low values of bias currents (order of $n A)$. This paper is an extension of work originally presented in [1].

The cutoff frequency $\left(f_{o}\right)$ of log-domain and $G_{m}-C$ filters is given by (1) [2, 3].

$$
f_{o}=\frac{G_{m}}{2 \pi C}=\frac{I_{o}}{2 \pi \eta V_{T} C}
$$

where $\eta, I_{o}$ and $V_{T}$ are sub-threshold slope factor, bias current and thermal voltage respectively. Equation (2) gives the expression for thermal voltage.

$$
V_{T}=\frac{k T}{q}
$$

where $k, T$ and $q$ have their usual meanings.

Equation (11) shows the direct relation between cutoff frequency and bias current. $I_{o}$ is usually generated from a current reference circuit. The current reference should be stable against variations in process and supply voltage. Supply voltage sensitivity is the parameter which shows the stability of the current reference against supply voltage variations. Lower supply voltage sensitivity indicates a better current reference. Depending on the mode of operation of transistors in the circuit or the topology of the filter architecture, the current reference can be a temperature independent or Proportional to Absolute Temperature (PTAT) current reference. From (1) and (2), in log-domain and $G_{m}-C$ filters, it is evident that $f_{o}$ has an inverse relationship with temperature. To fix this problem, $I_{o}$ is generated in such a way that it has also a linear relationship with temperature. Thus a PTAT current reference is used to bias $\log$-domain $/ G_{m}-C$ filters. As a cumulative effect, the filter cutoff frequency remains constant over temperature variations.

Many PTAT current reference designs are explored in the literature. Beta-multiplier based circuits discussed in [4]-[5] have adequate PTAT current characteristics. However, to generate low reference currents under low supply voltage for low frequency filters used in biological data acquisition system, larger values of resistors are needed which occupies large area on chip. Moreover the control over temperature coefficient of resistance also becomes

${ }^{*}$ Corresponding Author: Muhammed Mansoor C. B., Research scholar, Dept. of Electronics and Communication Engineering, National Institute of Technology Karnataka, Surathkal, India, +91 9497368483, mansoorcb@gmail.com 
difficult. A comprehensive research has been carried out on the conventional PTAT designs to tackle such shortcomings [6]-[7]. In [7], self-cascode transistors are used instead of resistor. This improves supply variation rejection, but linearity in current versus temperature characteristic has been compromised. In [8], composite transistors are used in the PTAT circuit to enhance the supply voltage sensitivity, but large resistor values are needed to generate smaller currents. In [2, 9], switched capacitors are used in place of resistor. But PTAT current is generated from PTAT voltage using opamp circuits like voltage to current converter which in turn increases the overall power dissipation of the circuit. [1] shows a good switched capacitor based PTAT reference. But the settling time of the reference current is compromised.

Settling time of current reference becomes a significant aspect especially in high speed applications. When the settling time of the reference current increases, the circuit which uses this reference current to generate its bias current also takes a significant amount of time to settle its bias current to steady state value. In case of a filter used in a wireless communication system, it takes a significant time to fix its cutoff frequency which may lead to attenuation of relevant signal information. For example, the circuit presented in [1] requires $8 \mathrm{~ms}$ to stabilise its current which means that it takes around $8 \mathrm{~ms}$ for the subsequent filter to fix its cutoff frequency which is biased by this reference current source. This work proposes a feasible solution to this problem.

In this paper, a fast settling PTAT current source is proposed. A startup circuit added to the PTAT source improves the settling time of reference current. The proposed circuit exhibits good PTAT characteristics and better stability against power supply fluctuations. This can be used to bias low frequency filters in biomedical applications. A low power log-domain filter is also designed and biased using the proposed PTAT current source. Results show that the cutoff frequency of the filter remains constant irrespective of temperature variations. This paper is organized as follows. Details of the proposed PTAT circuit along with the results are given in Section 2 Log-domain filter realisation and filter response are detailed in Section 3 Finally conclusions are drawn in Section 4

\section{Proposed Improved PTAT Reference}

A conventional beta-multiplier based current source circuit [5] is shown in Figure 1. Depending on the mode of operation of transistors, it can be a constant- $G_{m}$ biasing circuit [5] or a PTAT current source. It acts as former if all the transistors work in strong-inversion saturation region $\left(V_{G S}>V_{t h}\right.$ and $\left.V_{D S} \geq V_{G S}-V_{t h}\right)$ and as latter if all the transistors work in weak-inversion saturation $\left(V_{G S}<V_{t h}\right.$ and $V_{D S} \geq 3 V_{T}$ ).

When transistor operates in weak-inversion region, its drain current [10] can be expressed as following.

$$
I_{D}=\frac{W}{L} I_{D 0} e^{\frac{V_{G S}-V_{t h}}{\eta V_{T}}}\left(1-e^{\frac{-V_{D S}}{V_{T}}}\right)
$$

where $I_{D 0}=\mu_{n} C_{o x}(\eta-1) V_{T}^{2} ; \mu_{n}$ and $C_{o x}$ are electron mobility and gate oxide capacitance per unit area respectively. Other terms have their usual meanings. For $V_{D S} \geq 3 V_{T}$ (weak-inversion saturation region), (3) can be approximated to (4).

$$
I_{D}=\frac{W}{L} I_{D 0} e^{\frac{V_{G S}-V_{t h}}{\eta V_{T}}}
$$

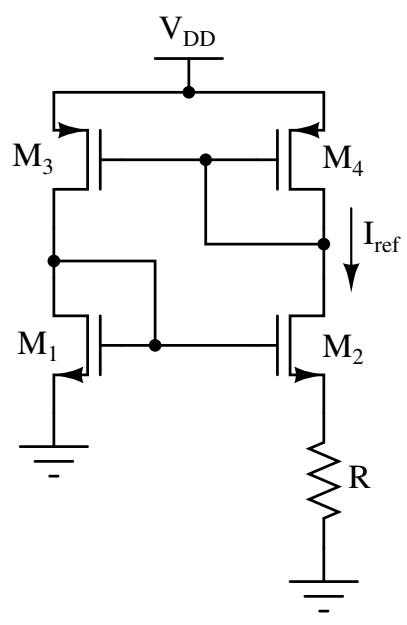

Figure 1: Beta-multiplier circuit

From Figure 1 the reference current $I_{r e f}$ which is also the drain current of $M_{2}\left(I_{D 2}\right)$ can be written as (5).

$$
I_{\text {ref }}=\frac{V_{R}}{R}
$$

Voltage across resistor $\mathrm{R}\left(V_{R}\right)$ can be given as (6).

$$
V_{R}=V_{G S 1}-V_{G S 2}
$$

Using (4), gate-source voltages of $M_{1}$ and $M_{2}$ can be expressed as (7) and (8) respectively.

$$
\begin{aligned}
& V_{G S 1}=\eta V_{T} \ln \left(\frac{I_{D 1}}{I_{D 0}\left(\frac{W}{L}\right)_{1}}\right)+V_{t h 1} \\
& V_{G S 2}=\eta V_{T} \ln \left(\frac{I_{D 2}}{I_{D 0}\left(\frac{W}{L}\right)_{2}}\right)+V_{t h 2}{ }^{\prime}
\end{aligned}
$$

$V_{t h 2}{ }^{\prime}$ can be written as 9] [11] to include the body effect of $M_{2}$.

$$
V_{t h 2^{\prime}}=V_{t h 2}+(\eta-1) V_{S B}=V_{t h 2}+(\eta-1)\left(V_{R}\right)
$$

By substituting (7) and (8) in (6) and by assuming $V_{t h 1}=V_{t h 2}, V_{R}$ can be derived as following.

$$
V_{R}=I_{r e f} \cdot R=V_{T} \ln (p)
$$

where

$$
p=\frac{(W / L)_{2}}{(W / L)_{1}}
$$

Thus, $I_{\text {ref }}$ can be expressed as 11 .

$$
I_{\text {ref }}=\frac{V_{T} \ln (p)}{R}
$$

Assuming $R$ is thermally stable, $I_{r e f}$ is directly proportional to temperature thereby giving PTAT characteristics. For lower values of currents (order of $n A \mathrm{~s}$ ), large values of resistors are needed. For example, to generate a reference current of $1 \mathrm{nA}$ using circuit shown in Figure 1 a $10 \mathrm{M} \Omega$ resistor is required if $p$ is chosen as 1.5. To accommodate such large resistors on chip, huge area is needed 
which makes the circuit more process dependent, hence reducing the accuracy of $I_{r e f}$. In a typical SoC (System-on-Chip), where digital circuits and analog circuits co-exist together, a stable and precise clock will be available. In such systems, the resistor $R$ in Figure 1 can be replaced by a switched capacitor driven by this clock which significantly enhances accuracy of $I_{r e f}$.

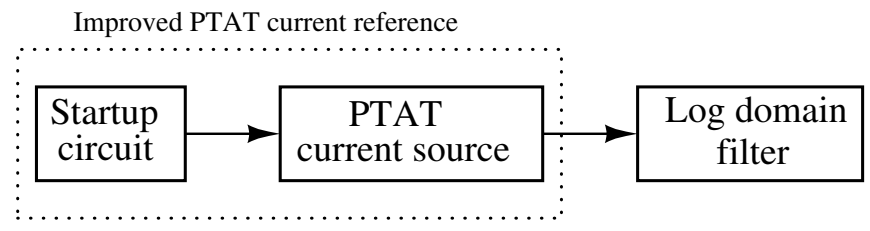

Figure 2: Block diagram

The entire block diagram of the circuit setup explained in this paper is shown in Figure 2. The startup circuit is added to the PTAT current generator to reduce the settling time of $I_{\text {ref }}$ thereby improving the transient response.

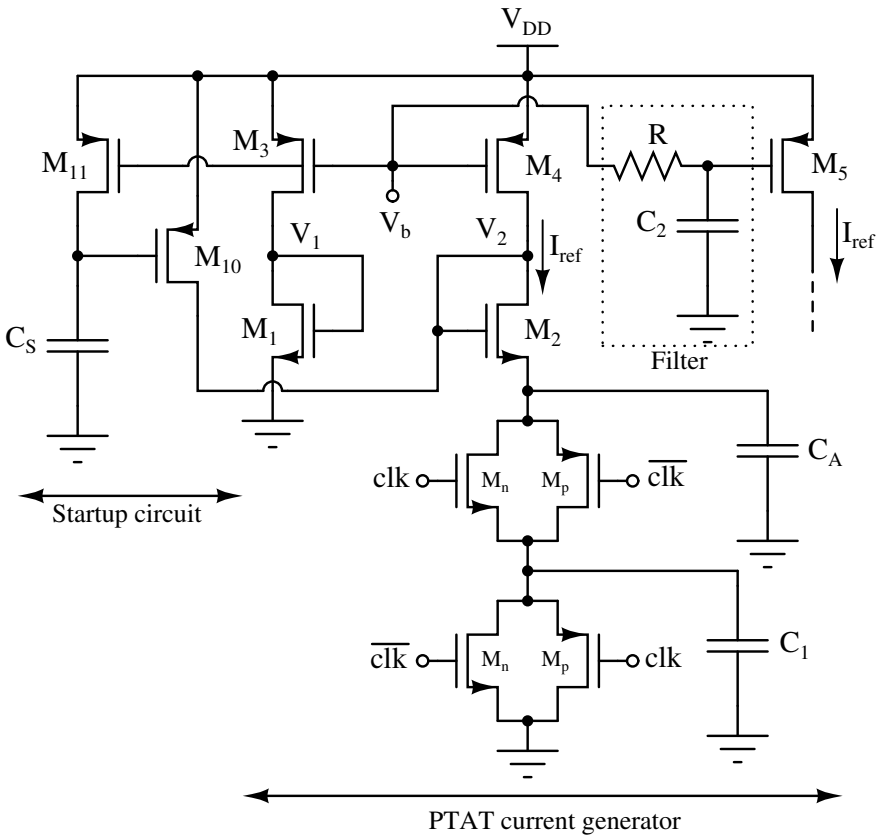

Figure 3: Proposed improved PTAT current reference

The transistor level implementation of PTAT current reference along with startup arrangement is shown in Figure 3 . In the proposed circuit, every transistor is working in weak-inversion region. The value of $p$ is chosen as 1.5 which means that the size of $M_{2}$ is 1.5 times the size of $M_{1}$. A switched capacitor comprising of capacitor $C_{1}$ and transmission gate switches $\left(M_{n}\right.$ and $\left.M_{p}\right)$ is used in place of resistor. $M_{p}$ is selected 4 times larger than $M_{n}$ in the transmission gate so that its on resistance does not depend on the voltage across it. Two clocks ('clk' and ' $\overline{c l k}$ ') which are complementary to each other and having frequency $f_{c l k}$ are used for the switching operation When 'clk' becomes high ('clk' becomes low), capacitor $C_{1}$ charges and when 'clk' becomes low (' $\overline{c l k}$ ' becomes high), it discharges. The average resistance $\left(R_{e q}\right)$ of the switched capacitor is given as in (12) [5].

$$
R_{e q}=\frac{1}{f_{c l k} C_{1}}
$$

By substituting $R_{e q}(12)$ in place of $R$ in $(11)$, we get $(13)$.

$$
I_{\text {ref }}=V_{T} f_{c l k} C_{1} \ln (p)
$$

From (13) it can be seen that, $I_{r e f}$ is directly proportional to $C_{1}$. If $f_{c l k}$ and $p$ are chosen properly, smaller capacitor values can be obtained for the currents in the order of $n A$.

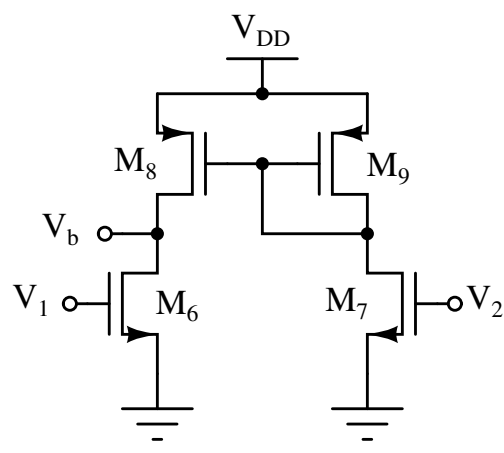

Figure 4: Error amplifier

The purpose of error amplifier which is shown in Figure 4 is to make the drain voltages of $M_{1}$ and $M_{2}$ equal thereby making currents through them equal. This kind of biasing minimises the dependency of $I_{r e f}$ on $V_{D D}$ which improves supply voltage sensitivity.

It takes around $8 \mathrm{~ms}$ for the reference current to settle to the steady state value of $1 \mathrm{nA}$ in the circuit presented in [1]. To reduce this delay, a startup arrangement comprising of $M_{10}, M_{11}$ and $C_{S}$ is added to the circuit. A similar kind of startup circuit is discussed in [12]. In Figure 3, when the circuit is switched on and before it reaches the steady state, initially the gates of $M_{1,2}$ and $M_{3,4}$ are at zero volt and $V_{D D}$ respectively. Assuming zero initial voltage across the capacitor $C_{S}, M_{10}$ turns on and pulls up the gates of $M_{1,2}$ to $V_{D D}$. $M_{1,2}$ start conducting and $I_{r e f}$ rises. Then, potential at the gates of $M_{3,4}$ drops and $M_{11}$ turns on. $C_{S}$ starts to charge towards $V_{D D}$ and potential at the gate of $M_{10}$ increases and once the circuit reaches steady state, $M_{10}$ turns off. $M_{10}$ and $M_{11}$ do not have a static current path from $V_{D D}$ to ground. Hence startup circuit dissipates zero static power.

Value of capacitor $C_{S}$ and sizes of $M_{10}$ and $M_{11}$ determine how fast startup circuit can initialise the main circuitry and how fast it can be disconnected once main circuitry reaches the steady state. Transistors $M_{10}$ and $M_{11}$ operate in linear region and the linear on resistance of these transistors are given by (14).

$$
R_{o n}=\frac{1}{\mu_{n} C_{o x} \frac{W}{L}\left(V_{G S}-V_{t h}\right)}
$$

How fast startup circuit is switched on depends on how fast transistor $M_{10}$ turns on. The on time of $M_{10}$ is limited by the input capacitance of $M_{10}$ which comprises of gate to source and gate to drain capacitances. By properly choosing the size of $M_{10}$, the on time can be optimised.

When it comes to disconnection of startup circuitry, assuming $M_{11}$ as a linear resistance given by (14), the RC time constant ( $\tau$ ) of the branch consisting of $M_{11}$ and $C_{S}$ is given by (15).

$$
\tau=\frac{C_{S}}{\mu_{n} C_{o x} \frac{W}{L}\left(V_{G S}-V_{t h}\right)}
$$


When capacitor $C_{S}$ charges above the voltage $\left(V_{D D}-V_{t h}\right), M_{10}$ turns off. $\left(V_{D D}-V_{t h}\right)$ is approximately $150 \mathrm{mV}$ in this design and time for $C_{S}$ to charge to this voltage ( $30 \%$ of final value) from $0.5 \mathrm{~V}$ supply through $M_{11}$ is nearly $0.4 \tau$.

A low pass filter which comprises of $R$ and $C_{2}$ is added to maintain the ripple content in the output reference current within the accepted level. Capacitor $C_{2}$ is chosen as $0.1 \mathrm{pF}$ and resistor $R$ is tuned to obtain the ripple content in $I_{\text {ref }}$ less than $1 \%$. To remove high frequency noise during switching operation, capacitor $C_{A}$ is used at the source of $M_{2}$. A $100 \mathrm{kHz}$ clock is selected for this work. The capacitors, $C_{1}, C_{A}, C_{S}$ are taken as $0.8 \mathrm{pF}, 10 \mathrm{pF}, 1 \mathrm{pF}$ respectively. By substituting (13) in (1), $f_{o}$ can be expressed as (16).

$$
f_{o}=\frac{f_{c l k} C_{1}}{2 \pi \eta C} \ln (p)
$$

From (16), it can be seen that $f_{o}$ is independent of temperature.

\subsection{Simulation results}

The proposed circuit is designed using UMC $65 \mathrm{~nm}$ CMOS process with a supply voltage of 0.5 V. Periodic Steady State (PSS) analysis is carried out to plot the reference current. Figure 5 shows the output current generated $(1 \mathrm{nA})$ for one clock period $(10 \mu \mathrm{s})$ at room temperature $\left(27^{\circ} \mathrm{C}\right)$.

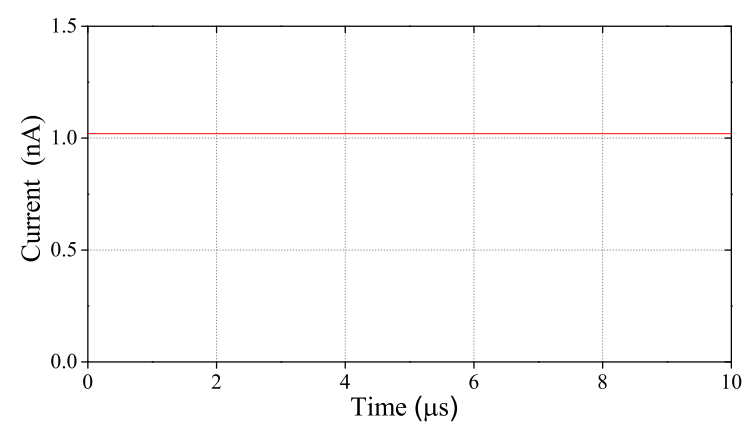

Figure 5: $I_{r e f}$ at $V_{D D}=0.5 \mathrm{~V}$

Figure 6 shows $I_{\text {ref }}$ variations when temperature is varied from $-10^{\circ} \mathrm{C}$ to $80^{\circ} \mathrm{C}$ for $V_{D D}=0.5 \mathrm{~V}$. It can be observed that $I_{r e f}$ changes linearly with temperature and thus has a good PTAT characteristics over the range of temperature.

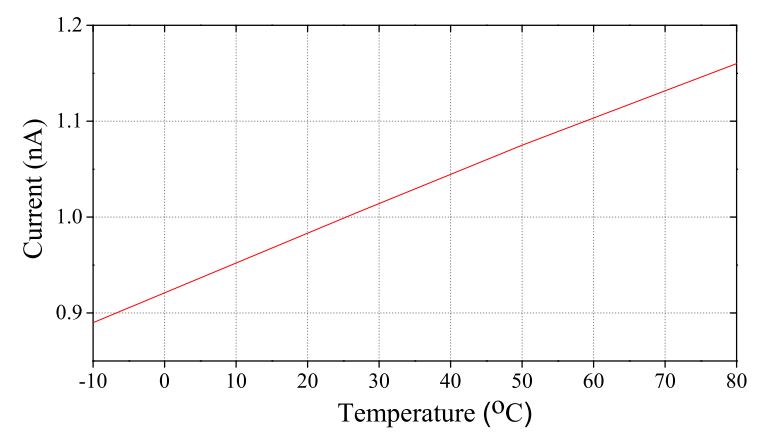

Figure 6: $I_{\text {ref }}$ vs. Temperature plot

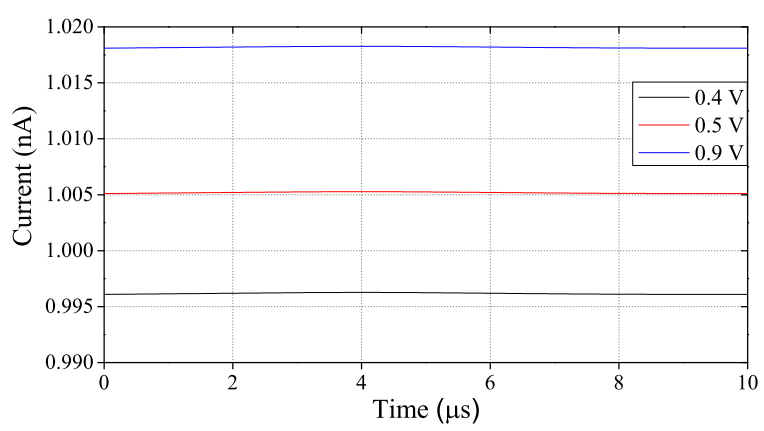

Figure 7: $I_{r e f}$ for different supply voltages

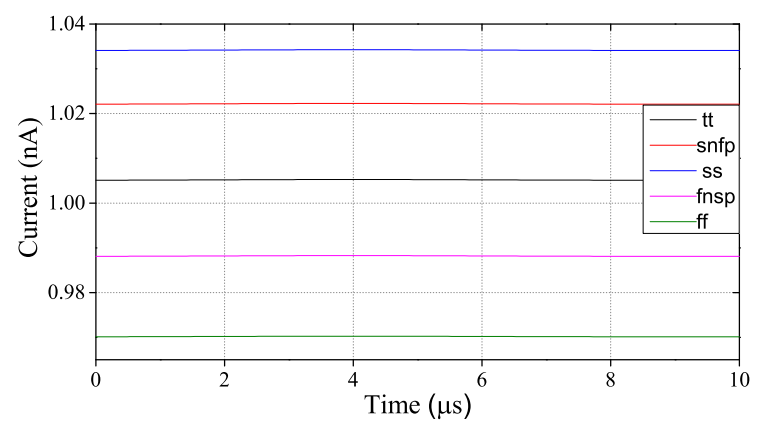

Figure 8: $I_{\text {ref }}$ across the process corners

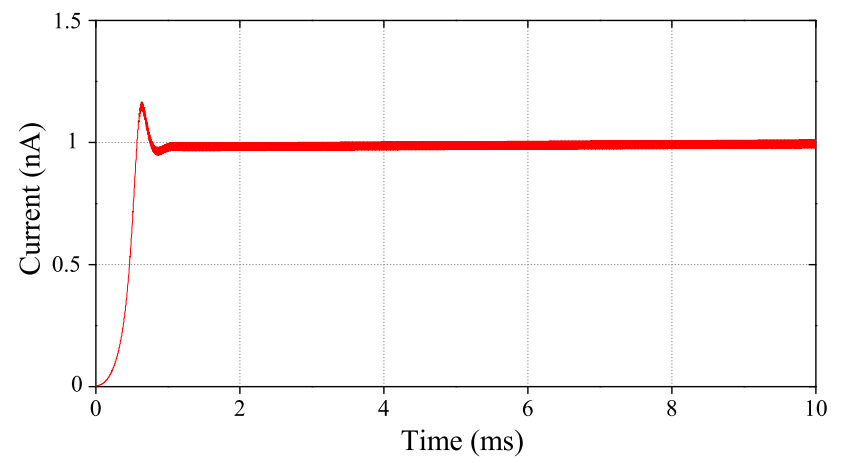

Figure 9: Transient response of the current flowing through $M_{2}$

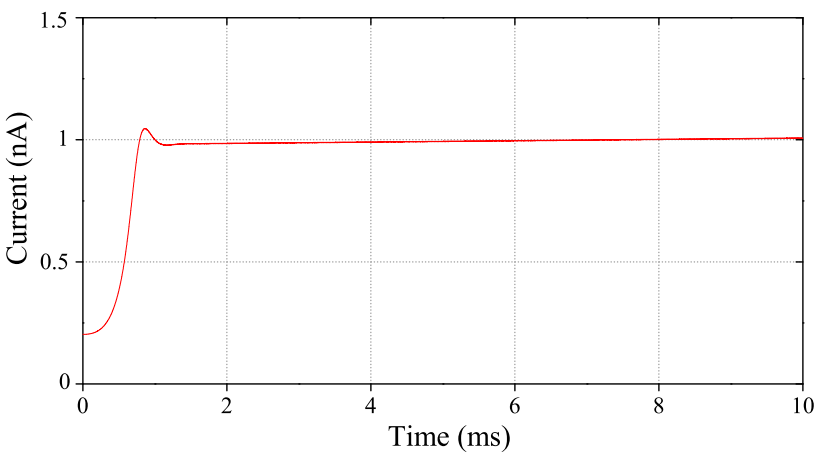

Figure 10: Transient response of the current flowing through $M_{5}$ 
$I_{\text {ref }}$ is plotted for different values of $V_{D D}$ at room temperature as shown in Figure 7. $I_{\text {ref }}$ is $0.996 n A$ and $1.018 n A$ for $V_{D D}$ of 0.4 $\mathrm{V}$ and $0.9 \mathrm{~V}$ respectively. The supply voltage sensitivity of $I_{r e f}$ is found to be $3.23 \% / V$ by considering these extreme values.

Figure 8 plots $I_{r e f}$ for various process corners at room temperature and $V_{D D}=0.5 \mathrm{~V}$. $I_{\text {ref }}$ deviates maximum in $s s$ and $f f$ corners $(1.034 n A$ and $0.968 n A)$. The maximum deviation is less than \pm 3.6 $\%$.

The current flowing through the source of $M_{2}$ is shown in Figure 9. It has ripples having peak to peak magnitude of approximately $0.1 \mathrm{nA}$. The ripple free current flowing through $M_{5}$ is shown in Figure 10 The settling time is found to be approximately $1 \mathrm{~ms}$.

Table 1: Comparison of the proposed work with other similar works

\begin{tabular}{|c|c|c|c|c|c|}
\hline & {$[\mathbf{7}$} & {$[\mathbf{1 3}$} & {$[\mathbf{1 4}$} & $\mathbf{[ 1}$ & $\begin{array}{c}\text { This } \\
\text { work }\end{array}$ \\
\hline Technology (nm) & 1500 & 180 & 40 & 65 & 65 \\
\hline Supply voltage (V) & 1.2 & 1.2 & 1 & 0.5 & 0.5 \\
\hline Reference current (nA) & 0.4 & 6 & 100 & 1 & 1 \\
\hline Settling time (ms) & - & - & - & 8 & 1 \\
\hline Power (nW) & 2 & - & 350 & 1.5 & 1.5 \\
\hline Supply voltage sensitivity (\%/V) & 6 & 6.47 & 2.9 & 2.7 & 3.2 \\
\hline
\end{tabular}

The improved PTAT reference consumes a power of $1.5 \mathrm{nW}$ at room temperature from a supply voltage of $0.5 \mathrm{~V}$. The proposed circuit along with existing PTAT current references in the literature are listed in Table 1 and a fair comparison is done. The proposed PTAT circuit functions under the lowest supply voltage and has a very low supply voltage sensitivity and settles faster than [1] (nearly $87 \%$ improvement in settling time).

\section{Log-Domain Filter Realization}

The proposed PTAT current source shows excellent PTAT characteristics. To verify this, a low frequency log-domain filter is designed and biased with the proposed switched capacitor based PTAT current reference. This log-domain filter can be used in low power biomedical applications like processing of ECG, EEG signals. The expectation is that the cutoff frequency of the filter remains the same irrespective of the variations in temperature.

The transfer function of a first order low-pass filter is

$$
H(s)=\frac{Y(s)}{X(s)}=\frac{\omega_{o}}{s+\omega_{o}}
$$

where $\omega_{0}$ is the cutoff frequency of the filter. The corresponding time domain differential equation is given by

$$
\frac{d y(t)}{d t}+\omega_{o} y(t)=\omega_{o} x(t)
$$

where $\mathrm{x}(\mathrm{t})$ and $\mathrm{y}(\mathrm{t})$ represent the input and output currents of the filter respectively. By solving the differential equations and relating the current-voltage in transistor operating in subthreshold operation, we get

$$
I_{K} I_{\text {out }}=I_{d c} I_{\text {in }}
$$

where $I_{\text {in }}$ contains both dc current $I_{d c}$ and input current $I_{\text {signal }}$. $I_{K}$ is the current flowing through $M_{3}$ which contains both capacitor current $I_{C}$ and $I_{d c}$. $I_{\text {out }}$ is the output current. Equation (19) can be realized using a translinear loop having four transistors. These equations can be implemented in the form of a translinear circuit as shown in Figure 11 [15].

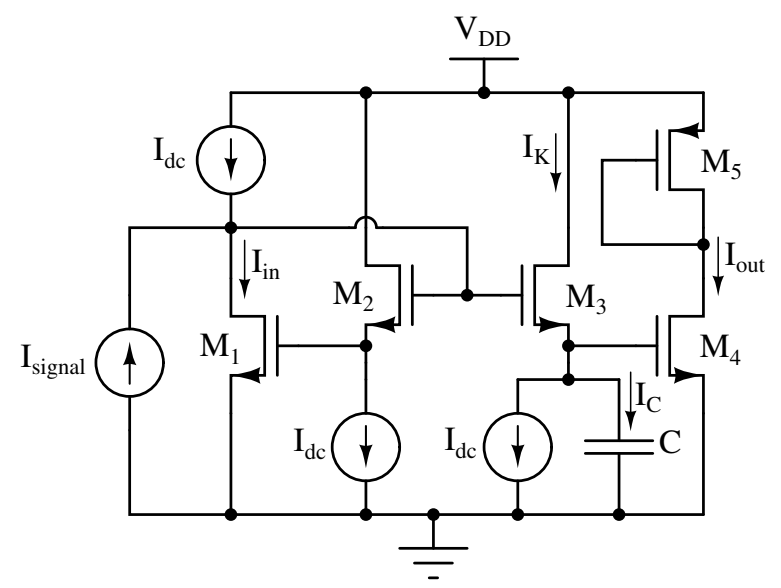

Figure 11: First order log-domain filter

In this circuit, the MOSFETs $M_{1}-M_{4}$ form the translinear loop. These transistors are working in sub threshold saturation region. The cutoff frequency of the filter can be tuned by varying the DC current and is given by following [16].

$$
f_{o}=\frac{I_{d c}}{2 \pi \eta V_{T} C}
$$

The ideal DC current sources shown in Figure 11 can be replaced by current sources implemented using MOSFETs. In this work $I_{d c}$ is taken as $I_{r e f}(1 \mathrm{nA})$. The cutoff frequency of $100 \mathrm{~Hz}$ is obtained by choosing $\mathrm{C}=37 \mathrm{pF}$.

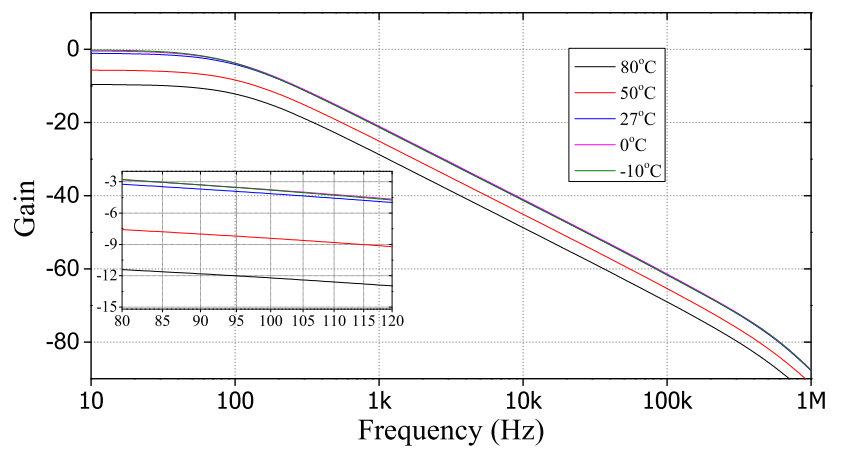

Figure 12: $\mathrm{AC}$ response of the filter biased with temperature independent current reference

\subsection{Simulation results}

The filter is designed using UMC $65 \mathrm{~nm}$ CMOS technology with a supply voltage of $0.5 \mathrm{~V}$. For a comparison, $I_{d c}$ is generated from a temperature independent current reference and ac response of the filter is plotted. Figure 12 shows the variation of cutoff frequency 
in the temperatures ranging from $-10^{\circ} \mathrm{C}$ to $80^{\circ} \mathrm{C}$. The maximum deviation is found to be $16 \%$. To verify the PTAT characteristics, $I_{d c}$ is generated from the proposed PTAT source and ac response is plotted. Figure 13 shows that deviation of cutoff frequency is negligible and maximum deviation is found to be less than $2 \%$. The band transitions are enlarged in corresponding inset graphs. Table 2 shows the deviation of filter cutoff frequency for both temperature independent and the proposed PTAT references.

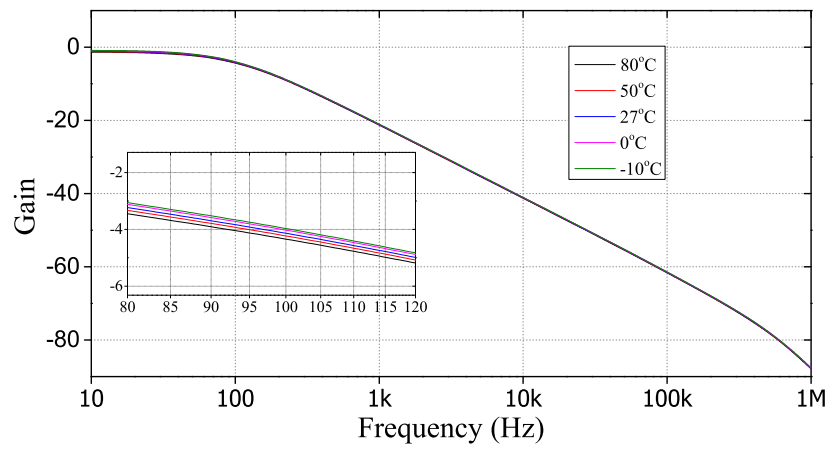

Figure 13: AC response of the filter biased with PTAT current reference

Table 2: Cutoff frequency deviation

\begin{tabular}{|c|c|c|}
\hline $\begin{array}{c}\text { Temperature } \\
\left({ }^{\circ} \mathbf{C}\right)\end{array}$ & \multicolumn{2}{|c|}{ Cutoff frequency (Hz) } \\
\cline { 2 - 3 } & Temp. independent & PTAT \\
\hline-10 & 116 & 101.9 \\
\hline 0 & 107 & 101.2 \\
\hline 27 & 100 & 100 \\
\hline 50 & 93 & 99 \\
\hline 80 & 88 & 98.4 \\
\hline
\end{tabular}

Table 3 lists the parameters of the filter designed in this work.

Table 3: Parameters of the filter

\begin{tabular}{|c|c|}
\hline Technology & $65 \mathrm{~nm} \mathrm{CMOS}$ \\
\hline Supply voltage & $0.5 \mathrm{~V}$ \\
\hline Power & $2.5 \mathrm{nW}$ \\
\hline Cutoff frequency & $100 \mathrm{~Hz}$ \\
\hline DC gain (at $27^{\circ} \mathrm{C}$ ) & 0.94 \\
\hline$I_{\text {in.pp }}$ at $25 \mathrm{~Hz}$ for $1 \%$ THD & $0.82 \mathrm{nA}$ \\
\hline Input referred noise from $1 \mathrm{~Hz}$ to $100 \mathrm{~Hz}$ & $3.26 \mathrm{pA}_{\mathrm{rms}}$ \\
\hline Dynamic range & $48 \mathrm{~dB}$ \\
\hline
\end{tabular}

\section{Conclusion}

This paper proposes a low voltage, low power, fast settling switched capacitor PTAT current reference circuit. Settling time is reduced with the aid of a startup circuit. The PTAT circuit exhibits good PTAT characteristics in the temperatures ranging from $-10^{\circ} \mathrm{C}$ to $80^{\circ} \mathrm{C}$ while operating under a very low power. The circuit is less susceptible to power supply fluctuations and can work at supply voltages as low as $0.4 \mathrm{~V}$. To test the PTAT source, a low power logdomain low frequency filter is also designed and biased with PTAT reference. Simulation results imply that the filter cutoff frequency remains unchanged irrespective of temperature variations and this validates the theory explained in the paper.

\section{References}

[1] H. G. Rao, S. Rekha, "A 0.5 V, 1 nA Switched Capacitor PTAT Current Reference Circuit," in 2019 IEEE Conference on Modeling of Systems Circuits and Devices (MOS-AK India), 51-54, 2019, doi:http://idr.nitk.ac.in/jspui/handle/ $123456789 / 7046$.

[2] A. Gerosa, A. Maniero, A. Neviani, "A fully integrated dual-channel logdomain programmable preamplifier and filter for an implantable cardiac pacemaker," IEEE Transactions on Circuits and Systems I: Regular Papers, 51(10), 1916-1925, 2004, doi:https://doi.org/10.1109/TCSI.2004.835027.

[3] R. Schaumann, H. Xiao, V. V. Mac, Design of Analog Filters 2nd Edition, Oxford University Press, Inc., 2009.

[4] E. Vittoz, J. Fellrath, "CMOS analog integrated circuits based on weak inversion operations," IEEE journal of solid-state circuits, 12(3), 224-231, 1977, doi:https://doi.org/10.1109/JSSC.1977.1050882.

[5] B. Razavi, Design of analog CMOS integrated circuits, Tata McGraw-Hill, 2002.

[6] L. Magnelli, F. Crupi, P. Corsonello, C. Pace, G. Iannaccone, "A 2.6 $\mathrm{nW}, 0.45 \mathrm{~V}$ temperature-compensated subthreshold CMOS voltage reference," IEEE Journal of Solid-State Circuits, 46(2), 465-474, 2011, doi: https://doi.org/10.1109/JSSC.2010.2092997.

[7] E. M. Camacho-Galeano, C. Galup-Montoro, M. C. Schneider, "A 2-nW 1.1-V self-biased current reference in CMOS technology," IEEE Transactions on Circuits and Systems II: Express Briefs, 52(2), 61-65, 2005, doi: 10.1016/j.trc.2016.07.007.

[8] H. G. Rao, C. B. M. Mansoor, S. Rekha, "A 0.8 V, 5 nA PTAT current reference circuit with improved supply voltage sensitivity," in 2019 Global Conference for Advancement in Technology (GCAT), 1-4, 2019.

[9] S. Malik, M. Schlarmann, R. Geiger, "A low temperature sensitivity switchedcapacitor current reference," in Proceedings of the European Conference on Circuit Theory and Design (ECCTD), 269-272, Citeseer, 2001.

[10] Y. Tsividis, C. McAndrew, Operation and Modeling of the MOS Transistor, Oxford Univ. Press, 2011.

[11] L. H. Ferreira, T. C. Pimenta, R. L. Moreno, "An ultra-low-voltage ultralow-power weak inversion composite MOS transistor: Concept and applications," IEICE transactions on electronics, 91(4), 662-665, 2008, doi: https://doi.org/10.1093/ietele/e91-c.4.662.

[12] S. Kumar, H. G. Rao, S. Rekha, "A 1-V, 8.6-nA Resistor-less PTAT Current Reference with Startup Circuit," in 2018 15th IEEE India Council International Conference (INDICON), 1-4, 2018.

[13] Y. Osaki, T. Hirose, N. Kuroki, M. Numa, "1.2-V supply, 100-nW, 1.09-V bandgap and $0.7-\mathrm{V}$ supply, $52.5 \mathrm{-nW}, 0.55-\mathrm{V}$ subbandgap reference circuits for nanowatt CMOS LSIs," IEEE Journal of Solid-State Circuits, 48(6), 15301538, 2013, doi:https://doi.org/10.1109/JSSC.2013.2252523.

[14] V. Jorge, A. L. Aita, "A 1-V PTAT current reference circuit with $0.05 \% / \mathrm{V}$ current sensitivity to VDD," in Proceedings of IEEE International Symposium on Circuits and Systems (ISCAS), 502-505, 2016.

[15] A. Patil, C. B. M. Mansoor, H. G. Rao, S. Rekha, "Low power log-domain filter," in 2019 IEEE 16th India Council International Conference (INDICON), $1-4,2019$.

[16] J. Mulder, W. A. Serdijn, A. C. van der Woerd, A. H. van Roermund, Dynamic translinear and log-domain circuits: analysis and synthesis, volume 481, Springer Science \& Business Media, 2012. 